\title{
From nuclear clusters to halo globulars: Star clusters as basic galactic building blocks
}

\author{
Richard de Grijs \\ Department of Physics \& Astronomy, The University of Sheffield, Hicks Building, Hounsfield \\ Road, Sheffield S3 7RH, UK
}

\begin{abstract}
I assess the similarities and differences between the star-formation modes in quiescent spiral galaxies versus those in violent starburst regions, including galactic nuclei. As opposed to the quiescent star-formation mode, current empirical evidence on the star-formation processes in the extreme, high-pressure environments induced by galaxy encounters strongly suggests that star cluster formation is an important and perhaps even the dominant mode of star formation in such starburst events. This implies that by using star clusters as unique diagnostic probes, we can trace a galaxy's most violent star formation history very well, at least for the past few Gyr. The sizes, luminosities, and mass estimates of the young massive star clusters are entirely consistent with what is expected for young Milky Way-type globular clusters (GCs). Recent evidence lends support to the scenario that GCs, which were once thought to be the oldest building blocks of galaxies, are still forming today.
\end{abstract}

\section{Young massive star clusters as proto-globular clusters}

Luminous, massive yet compact star clusters (YMCs; often with masses $m_{\mathrm{cl}} \geqslant 10^{5} \mathrm{M}_{\odot}$ ) are the hallmarks of the most intense starbursts. YMCs are therefore important as benchmarks of cluster formation and evolution, and also as important tracers of the (stellar) initial mass function (IMF) and other physical properties of starbursts. This is so, because star clusters can extremely well be represented by single-age, single-metallicity "simple" stellar populations (SSPs). This sets the YMCs formed in violent starburst events apart from the apparently similar nuclear star clusters, since the latter are often characterised by stellar population mixes (e.g., Walcher et al. 2006). The defining characteristics of YMCs have been explored in starburst regions in several dozen galaxies, both in normal spirals and in gravitationally interacting galaxies.

The crucial question remains, however, whether or not at least some of the YMCs observed in extragalactic starbursts might survive to become (possibly somewhat more metal-rich) counterparts of the Galactic globular clusters (GCs) when they reach a similar age. If we could resolve this issue convincingly, one way or the other, the implications would be far-reaching for a wide range of astrophysical questions, including our understanding of how galaxy formation, assembly and evolution proceeds, and what the process and requisite conditions are for star (cluster) formation.

The evolution of young clusters depends crucially on their stellar IMF: if its slope is too shallow, i.e., if the clusters are significantly deficient in low-mass stars compared to, e.g., the solar neighbourhood, they will likely disperse within about a Gyr of their formation (e.g., Gnedin \& Ostriker 1997; Goodwin 1997; Smith \& Gallagher 2001; Mengel et al. 2002; de Grijs \& Goodwin, in prep.). At present, there are two principal approaches which one can use to address the underlying IMFs of extragalactic YMCs. 


\subsection{The Cluster Luminosity Function: the case of M82}

In de Grijs et al. (2003a,b) we reported the discovery of an approximately log-normal cluster luminosity and mass function (CLF, CMF) for the subpopulation of approximately coeval star clusters at the intermediate age of $\sim 1$ Gyr in M82's fossil starburst region "B". This provided the first deep CLF (CMF) for a star cluster population at intermediate age, which thus serves as an important benchmark for theories of the evolution of star cluster systems [see also Goudfrooij et al. (2004) for a related important result for NGC 1316, at $\sim 3 \mathrm{Gyr}$. The fact that we considered an approximately coeval subset of the M82 B cluster population, combined with our use of the 100 per cent completeness limit as our base line ensures the robustness of the CMF peak detection. Additional arguments in favour of this robustness are provided in de Grijs et al. (2005).

Most analyses of the young Large Magellanic Cloud cluster system (with ages $\leqslant 2 \times 10^{9}$ yr), starting with the seminal work by Elson \& Fall (1985), seem to imply that the CLF of YMCs is well described by a power law. However, in de Grijs \& Anders (2006) we showed that this power-law behaviour breaks down below $m_{\mathrm{cl}} \simeq 10^{3} \mathrm{M}_{\odot}$, as well as for our youngest LMC cluster age bins. Although the latter could partially be due to the so-called "infant mortality" effect, by which some $60-90$ per cent of newly formed clusters are disrupted in the first $\sim 10 \mathrm{Myr}$ of their lifetime (e.g., Bastian et al. 2005), the deviation of the LMC CLFs at low cluster masses is significant for ages up to at least 1 Gyr, beyond which our observational completeness limit prevents us from making definite statements on the CLF shape at low mass (de Grijs \& Anders 2006).

Nevertheless, for old GC systems with ages $\geqslant 10^{10} \mathrm{yr}$, the CLF shape is - on the other hand - well established to be roughly log-normal, and almost universal among local galaxies (barring a slight metallicity dependence). This type of observational evidence has led to the popular, but thus far mostly speculative theoretical prediction that not only a power-law, but any initial CLF (CMF) will be rapidly transformed into a lognormal distribution because of (i) stellar evolutionary fading of the lowest-luminosity (mass) objects to below the detection limit; and (ii) disruption of the low-mass clusters due both to interactions with the gravitational field of the host galaxy, and to internal two-body relaxation effects leading to enhanced cluster evaporation (but see Parmentier \& Gilmore 2006).

From our detailed analysis of the expected evolution of CMFs starting from initial log-normal and initial power-law distributions (de Grijs et al. 2005), we conclude that the observed turnover in the M82 B CMF is inconsistent with a scenario in which the 1 Gyr-old cluster population originated from an initial power-law mass distribution. This applies to a large range of "characteristic" cluster disruption time-scales, and is supported by arguments related to the initial density in M82 B, which would be unphysically high if the present cluster population were the remains of an initial power-law distribution (particularly in view of the effects of the cluster "infant mortality", which requires large excesses of low-mass unbound clusters to be present at the earliest times).

In de Grijs et al. (2003c) we showed that the CMFs of YMCs in many different environments are well approximated by power laws with slopes $\alpha \simeq-2$. However, except for the intermediate-age cluster systems in M82 B and NGC 1316 (Goudfrooij et al. 2004), the expected turn-over (or peak) mass (based on comparisons with present-day GC systems and taking evolutionary fading into account) in most YMC systems observed to date occurs close to or below the observational detection limit, simply because of their greater distances and shallower observations. As such, these results are not necessarily at odds with each other, but merely hindered by observational selection effects. 


\subsection{High-resolution spectroscopy: individual cluster analysis}

With the ever increasing number of large-aperture ground-based telescopes equipped with state-of-the-art high-resolution spectrographs and the wealth of observational data provided by the Hubble Space Telescope, we may now finally be getting close to resolving the potentially far-reaching issue of YMC-to-GC evolution conclusively. To do so, one needs to obtain (i) high-resolution spectroscopy, in order to obtain dynamical mass estimates, and (ii) high-resolution imaging to measure their sizes (and luminosities). As a simple first approach, one could then construct diagnostic diagrams of YMC mass-to-light ratio vs. age, and compare the YMC locations in this diagram with SSP models using a variety of IMF descriptions (cf. Smith \& Gallagher 2001; Mengel et al. 2002; Bastian et al. 2006). However, such an approach, while instructive, has serious shortcomings:

(i) In this simple approach, the data can be described by both variations in the IMF slope and variations in a possible low-mass cut-off; the models are fundamentally degenerate for these parameters.

(ii) While the assumption that these objects are approximately in virial equilibrium is probably justified at ages greater than a few $\times 10^{7}$ yr (at least for the stars dominating the light), the central velocity dispersion (as derived from luminosity-weighted highresolution spectroscopy) does not necessarily represent a YMC's total mass. It is now wellestablished that almost every YMC exhibits significant mass segregation from very early on, so that the effects of mass segregation must be taken into account when converting central velocity dispersions into dynamical mass estimates (see also Lamers et al. 2006; Fleck et al. 2006; Moll et al. 2006 and in prep.).

(iii) With the exception of a few studies (e.g., M82-F; Smith \& Gallagher 2001), the majority of YMCs thus far analysed in this way have ages around $10 \mathrm{Myr}$. Around this age, however, red supergiants (RSGs) appear in realistic stellar populations. Unfortunately, the model descriptions of the RSG phase differ significantly among the various leading groups producing theoretical stellar population synthesis codes (Padova vs. Geneva vs. Yale), and therefore the uncertainties in the evolutionary tracks are substantial.

(iv) For nuclear star clusters, one also needs to relax the SSP approximation, and allow multiple stellar populations to be present in the mix; this has the potential of matching the observed high-resolution spectra very well, however (Walcher et al. 2006).

\section{Where does this leave us?}

It may appear that a fair fraction of the $\sim 10$ Myr-old YMCs that have been analysed thus far may be characterised by unusual IMFs, since their loci in the diagnostic diagram are far removed from any of the "standard" SSP models (see, e.g., Bastian et al. 2006; Moll et al. 2006 and in prep.). However, Bastian \& Goodwin (2006) recently showed that this is most likely an effect of the fact that the velocity dispersions of these young bjects do not adequately trace their masses. They are instead strongly affected by the effects of gas expulsion due to supernova activity and massive stellar winds. In this respect, it is encouraging to see that the older clusters (i.e., older than M82-F, a few $\times 10^{7} \mathrm{yr}$ ) seem to conform to "normal" IMFs; by those ages, the clusters' velocity dispersions seem to represent the underlying gravitational potential much more closely.

We recently reported the discovery of a extremely massive, but old (12.4 $\pm 3.2 \mathrm{Gyr})$ GC in M31, 037-B327, that has all the characteristics of having been an exemplary YMC at earlier times (Ma et al. 2006). To have survived for a Hubble time, we conclude that its stellar IMF cannot have been top-heavy, i.e., characterized by a low-mass cut-off at $m_{\star} \geqslant 1 \mathrm{M}_{\odot}$, as sometimes advocated for current YMCs (e.g., Smith \& Gallagher 2001). 
Using this constraint, and a variety of SSP models, we determine a photometric mass for 037-B327 of $M_{\mathrm{GC}}=(3.0 \pm 0.5) \times 10^{7} \mathrm{M}_{\odot}$, somewhat depending on the SSP models used, the metallicity and age adopted and the IMF representation. In view of the large number of free parameters, the uncertainty in our photometric mass estimate is surprisingly small. This mass, and its relatively small uncertainties, make this object the most massive star cluster of any age in the Local Group. As a surviving "super" star cluster, this object is of prime importance for theories aimed at describing massive star cluster evolution.

\section{References}

Bastian N., Gieles M., Lamers H.J.G.L.M., Scheepmaker R., \& de Grijs R., 2005, A\&A, 431, 905 Bastian N., Saglia R.P., Goudfrooij P., Kissler-Patig M., Maraston C., Schweizer F., \& Zoccali M., 2006, A\&A, 448, 881

Bastian N., \& Goodwin S.P., 2006, MNRAS, 369, L9

de Grijs R., Bastian N., \& Lamers H.J.G.L.M., 2003a, ApJ, 583, L17

de Grijs R., Bastian N., \& Lamers H.J.G.L.M., 2003b, MNRAS, 340, 197

de Grijs R., Anders P., Lynds R., Bastian N., Lamers H.J.G.L.M., \& O'Neill E.J., Jr., 2003, MNRAS, 343, 1285

de Grijs R., Parmentier G., \& Lamers H.J.G.L.M., 2005, MNRAS, 364, 1054

de Grijs R., \& Anders P., 2006, MNRAS, 366, 295

Elson R.A.W., \& Fall S.M., 1985, PASP, 97, 692

Fleck J.J., Boily C.M.; Lançon A., \& Deiters S., 2006, MNRAS, 369, 1392

Gnedin O.Y., \& Ostriker J.P., 1997, ApJ, 474, 223

Goodwin S.P., 1997, MNRAS, 286, 669

Goudfrooij P., Gilmore D., Whitmore B.C., \& Schweizer F., 2004, ApJ, 613, L121

Lamers H.J.G.L.M., Anders P., \& de Grijs R., 2006, A\&A, 452, 131

Ma J., de Grijs R., Yang Y., Zhou X., Chen J., Jiang Z., Wu Z., \& Wu J., 2006, MNRAS, 368, 1443

Mengel S., Lehnert M.D., Thatte N., \& Genzel R., 2002, A\&A, 383, 137

Moll S.L., de Grijs R., Mengel S., \& Smith L.J., 2006, in: "Mass loss from stars and the evolution of stellar clusters", de Koter A., Smith L.J., Waters R., eds., (San Francisco: ASP), in press

Parmentier G., \& Gilmore G.F., 2006, MNRAS, submitted

Smith L.J., \& Gallagher J.S., 2001, MNRAS, 326, 1027

Walcher C.-J., Böker T., Charlot S., Ho L.C., Rix H.-W., Rossa J., Shields J.C., \& van der Marel R.P., 2006, ApJ, in press (astro-ph/0604138)

\section{Discussion}

EMSELLEM: Could the "super" stellar cluster you studied be the remnant nucleus of a galaxy that has been stripped?

DE GRIJS: Although this cannot be ruled out completely at present, I suspect that it really is a genuine GC. In addition to the fact that its SED is very well fit by a simple stellar population, it has a very small size $\left(R_{\mathrm{hl}} \sim 2.5 \pm 0.2 \mathrm{pc}\right.$ at the distance of M31, $m-M=24.88 \mathrm{mag}$ ), and shows no evidence of an extended envelope (Ma et al. 2006).

BLOCK: Does your LMC cluster sample span all the ages contained in the Searle, Wilkinson \& Bagnuolo cluster classification types I - VII?

DE GRIJs: Yes, it does. I refer you to Fig. 7 in de Grijs \& Anders (2006), where we show our full LMC sample in $\log$ (age)- $\log$ (mass) space, including the 50 per cent completeness limit. As you will notice, our LMC sample ranges from the youngest clusters (returned as $4 \mathrm{Myr}$ because of limitations of our models at young ages) to the well-known old GCs, with ages $>10$ Gyr. 